\title{
Upper tract urothelial carcinomas: frequency of association with mismatch repair protein loss and lynch syndrome
}

\author{
Holly L Harper ${ }^{1}$, Jesse K McKenney ${ }^{1,3}$, Brandie Heald ${ }^{2}$, Andrew Stephenson ${ }^{3}$, \\ Steven C Campbell ${ }^{3}$, Thomas Plesec ${ }^{1}$ and Cristina Magi-Galluzzi ${ }^{1,3}$ \\ ${ }^{1}$ Robert J. Tomsich Pathology and Laboratory Medicine Institute, Cleveland Clinic, Cleveland, OH, USA; \\ ${ }^{2}$ Genomic Medicine Institute, Cleveland Clinic, Cleveland, OH, USA and ${ }^{3}$ Glickman Urological Institute, \\ Cleveland Clinic, Cleveland, OH, USA
}

\begin{abstract}
Increased risk for upper tract urothelial carcinoma is described in patients with Lynch syndrome, caused by germline mutations in mismatch repair genes. We aimed to identify the frequency of mismatch repair protein loss in upper tract urothelial carcinoma and its potential for identifying an association with Lynch syndrome. We queried our database to identify upper tract urothelial carcinomas. Patients were cross-referenced for history of colorectal carcinoma or other common Lynch syndrome-associated neoplasms to enrich for potential Lynch syndrome cases. Tumor histopathologic characteristics were reviewed and each case was analyzed for loss of mismatch repair proteins, MLH1, MSH2, MSH6, and PMS2, by immunohistochemistry. Of 444 patients with upper tract urothelial carcinoma, a subset of 215 (encompassing 30 with upper tract urothelial carcinoma and another common Lynch syndrome-associated neoplasm) was analyzed for loss of mismatch repair protein expression. Of 30 patients with Lynch syndrome-associated neoplasms, six had documented Lynch syndrome, including two with Muir-Torre syndrome. Mismatch repair protein loss was identified in $7 \%$ of total upper tract urothelial carcinomas and $\mathbf{3 0} \%$ of patients with Lynch syndrome-associated neoplasms (including all patients with Lynch syndrome/Muir-Torre syndrome). Of patients without history of Lynch syndrome-associated neoplasms, 5 of 184 $(2.7 \%)$ had loss of mismatch repair protein expression. Twelve cases with mismatch repair protein loss demonstrated loss of MSH2 and MSH6, and 2 had isolated loss of MSH6. MLH1 and PMS2 expression were consistently retained. Although increased intratumoral lymphocytes, inverted growth, pushing tumor-stromal interface, and lack of nuclear pleomorphism were more commonly seen in cases with mismatch repair protein loss, only intratumoral lymphocytes and presence of pushing borders were statistically significant. MLH1 and PMS2 testing appear to have little utility in upper tract urothelial carcinoma; however, mismatch repair protein loss of MSH2 and/or MSH6 by immunohistochemistry seems relatively sensitive and specific for identifying patients with potential Lynch syndrome.
\end{abstract}

Modern Pathology (2017) 30, 146-156; doi:10.1038/modpathol.2016.171; published online 7 October 2016

Urothelial carcinoma is the fourth most common cancer occurring in males in the United States and is estimated to account for $4-5 \%$ of male cancer-related deaths. ${ }^{1}$ However, urothelial carcinoma occurring in the upper urinary tract, that is, in the renal pelvicaliceal system and/or ureter, has an annual incidence of 1-2 cases per 100000 people and

Correspondence: Professor C Magi-Galluzzi, MD, PhD, Robert J. Tomsich Pathology and Laboratory Medicine Institute, Cleveland Clinic, 9500 Euclid Avenue, L25, Cleveland, OH 44195, USA.

E-mail: magic@ccf.org

Received 6 June 2016; revised 16 August 2016; accepted 30 August 2016; published online 7 October 2016 accounts for only about $5-7 \%$ of all urothelial carcinomas. ${ }^{2-4}$ Compared with bladder urothelial carcinoma, patients with upper tract urothelial carcinoma tend to present with higher grade and higher stage disease, which portend a worse overall prognosis. ${ }^{5,6}$ The five-year cancer specific survival of upper tract urothelial carcinoma is greater than $90 \%$ for stage $\mathrm{Ta}$ or $\mathrm{T} 1$ disease, $<60 \%$ for $\mathrm{T} 2$ or $\mathrm{T} 3$ disease, and less than $10 \%$ for patients with $\mathrm{T} 4$ or metastatic (N/M1) tumors. ${ }^{7,8}$ Although some studies suggest that patients with low-risk disease can be managed conservatively, ${ }^{6,9,10}$ most patients with upper tract urothelial carcinoma are treated with nephroureterectomy or segmental ureterectomy. 
Lynch syndrome is an autosomal dominant hereditary tumor syndrome caused by germline mutations in genes encoding for mismatch repair proteins, which lead to microsatellite instability and increased risk of multiple tumor types. ${ }^{11,12}$ The mismatch repair complex is critical in the recognition and repair of nucleotide bases that have been erroneously inserted during DNA replication. Microsatellites are short, polymorphic tandem repeats dispersed throughout the genome that are particularly sensitive to mutability in patients with mismatch repair defects. ${ }^{13,14}$ A germline mutation in one of the major mismatch repair genes-MLH1, MSH2, MSH6, $P M S 2$, and EPCAM - can be identified in 90\% of all Lynch syndrome patients. ${ }^{15-17}$ Mutations in MSH2 account for $60 \%$ of cases and MLH1 for $30 \%$, whereas MSH6 (5-8\%) and PMS2 make up much of the remainder. ${ }^{18}$ More recent studies have identified mutations in the EPCAM gene, which lead to inactivation of $M S H 2,{ }^{16,19-21}$ or inversions in the MSH2 gene $\mathrm{e}^{22,23}$ as the causative abnormality in some patients; however, the specific underlying mutation remains unknown in a small percentage of cases. ${ }^{16,24}$ Carcinogenesis in these patients is thought to be due to an accumulation of somatic frameshift mutations within microsatellite regions of various genes controlling growth and apoptosis. ${ }^{15}$ These target genes appear to be tissue-specific and may account for the spectrum of disease seen in Lynch syndrome patients. ${ }^{25-27}$

Lynch syndrome is the most common hereditary cause of colorectal carcinoma, accounting for $2-3 \%$ of all colorectal carcinomas. ${ }^{16}$ Although colorectal carcinoma and endometrial carcinoma are the most frequently associated neoplasms, other common Lynch syndrome-associated neoplasms include gastric and small bowel carcinomas, hepatobiliary carcinomas, ovarian carcinomas, central nervous system neoplasms and urothelial carcinomas, specifically of the upper urinary tract. ${ }^{21,28}$ Prostate and testicular germ cell tumors have also been rarely reported in Lynch syndrome kindreds. ${ }^{21,29}$ Muir-Torre syndrome, which is considered a variant of Lynch syndrome, includes patients with sebaceous neoplasms or keratoacanthomas of the skin. The majority of Muir-Torre syndrome patients have MSH2 mutations; colorectal carcinoma and upper tract urothelial carcinoma are the most common visceral malignancies identified in these patients. ${ }^{20,30,31}$

Upper tract urothelial carcinoma occurs in 5\% of patients with Lynch syndrome, making it the third most common malignancy in this patient population..$^{5,13,32}$ Patients with Lynch syndrome have a lifetime risk of developing upper tract urothelial carcinoma of up to $20 \%,{ }^{33}$ with the highest risk among patients with MSH2 mutations. ${ }^{17,28,33-35}$ Lynch syndrome patients with upper tract urothelial carcinoma present at a younger age and often lack the typical risk factors associated with urothelial carcinoma in the general population. ${ }^{29,36}$ Studies have linked inverted growth pattern and low tumor stage in upper tract urothelial carcinoma to microsatellite instability, ${ }^{14,27,37}$ but other tumor characteristics, such as histologic grade and location, have shown variable results. Data regarding the risk of bladder urothelial carcinoma in Lynch syndrome as a whole are conflicting; ${ }^{38,39}$ however, patients with $\mathrm{MSH} 2$ mutations are at particular risk for the development of urothelial carcinoma, including carcinoma arising in the bladder. ${ }^{17,33,40,41}$

We evaluated the incidence of loss of mismatch repair protein expression by immunohistochemistry within a large cohort of upper tract urothelial carcinoma patients, enriched for cases with common Lynch syndrome-associated neoplasms (particularly colorectal carcinoma and endometrial carcinoma), to assess the utility of immunohistochemical studies targeting mismatch repair proteins in identifying patients with mismatch repair defects. As morphologic features characteristic of high microsatellite instability have been well documented in colorectal, ${ }^{16,42,43}$ endometrial, ${ }^{44-46}$ and ovarian carcinomas, ${ }^{47}$ we also studied similar features to determine which upper tract urothelial carcinoma patients might benefit from additional mismatch repair testing.

\section{Materials and methods}

A natural language search of the Cleveland Clinic pathology database (CoPath Plus) was performed to identify cases of upper tract urothelial carcinoma excised between 1995 and 2014. To maintain the purity of our series as papillary and/or invasive carcinomas arising within the upper tract, cases of flat carcinoma in situ and possible cases of involvement of the upper tract by a carcinoma of bladder origin were excluded. The patients' medical record numbers were cross-referenced with the Cleveland Clinic Lynch Syndrome Registry to identify study patients with a clinical diagnosis of Lynch syndrome. Medical record numbers were also crossreferenced with a second database of patients with colorectal adenocarcinoma (also retrieved via CoPath Plus natural language search), and all electronic medical charts were reviewed to identify patients with history of other common Lynch syndrome-associated neoplasms, particularly colorectal carcinoma or endometrial carcinoma, and Muir-Torre syndrome to enrich for potential Lynch syndrome patients.

For each case included in the study, all hematoxylin and eosin-stained (H\&E) sections of tumor were reviewed and the following histopathologic characteristics recorded (Figure 1): histologic grade; growth pattern (papillary, papillary/endophytic, flat, solid/ nodular); presence, type (pushing border vs infiltrative) and extent of invasion; presence, extent and composition of peri-tumoral inflammation; presence and extent of intratumoral lymphocytes; presence and extent of necrosis; presence of nuclear pleomorphism, apoptosis, and stromal desmoplasia. 

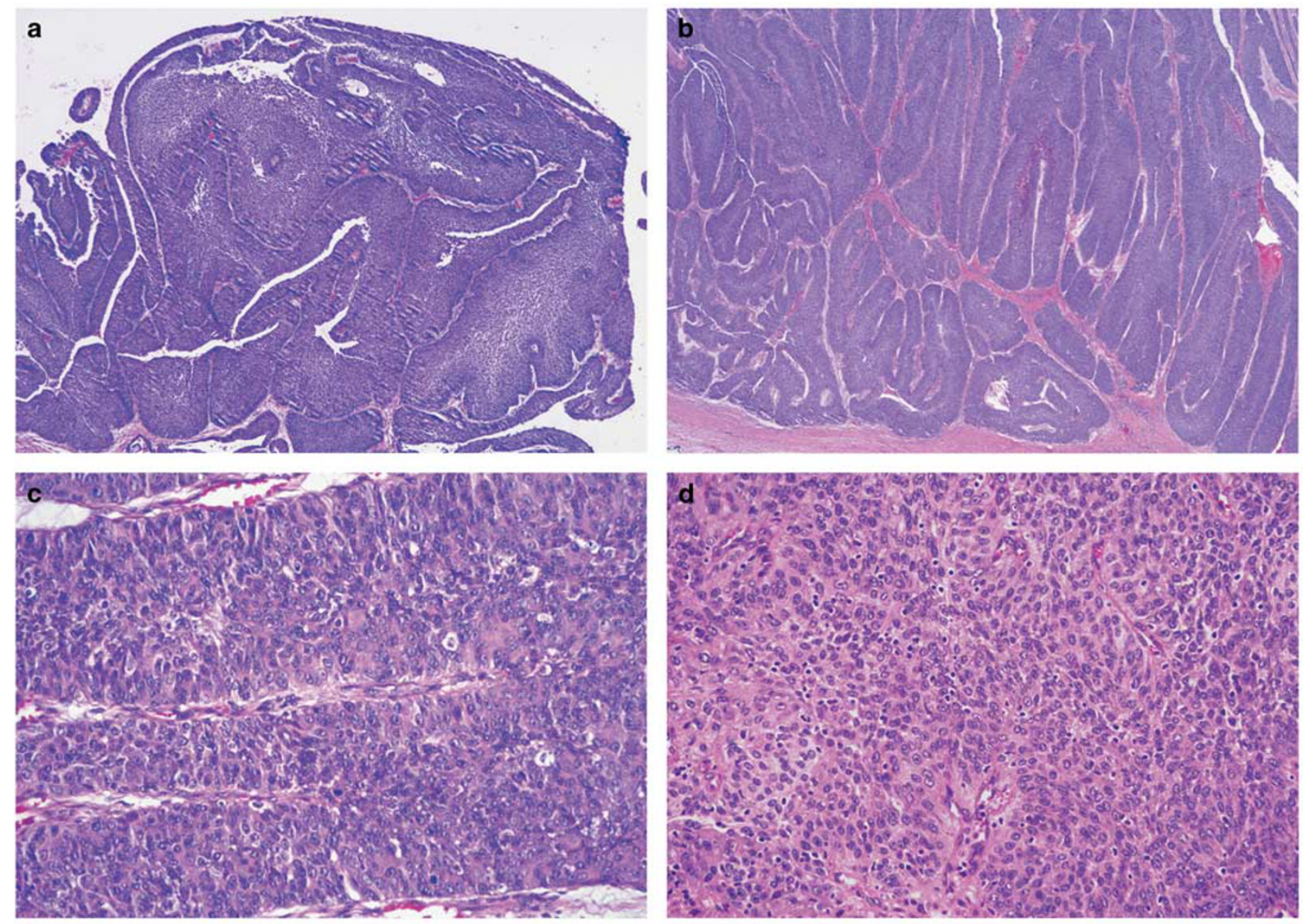

Figure 1 Common characteristics of tumors with mismatch repair protein loss by immunohistochemistry included inverted growth (a) or pushing borders without infiltration (b), high nuclear grade without significant pleomorphism (c), and moderate (2+) to marked (3+) intratumoral lymphocytic response (d).

Histologic grade of the non-invasive component was assigned based on the 2004 ISUP/WHO two-tiered grading scheme of low- and high-grade. ${ }^{48}$ Occasional tumors showed predominantly low-grade morphology with focal high-grade areas accounting for $<5 \%$ of the tumor and were recorded as low-grade with focal high-grade features. Significant nuclear pleomorphism was defined as pleomorphism meeting the threshold of G3 in the WHO 1973 classification system. ${ }^{49}$ Pushing border invasion was defined as a broad-based front with displacement of normal structures but without destructive infiltration of the tissue as nests or single cells. Peri-tumoral inflammation was considered $1+$ if it was appreciable at low magnification but was restricted to the tumor base, 2+ if inflammation was also present within the papillary stalks of the tumor and $3+$ if the inflammatory cells expanded the papillary stalks. Intratumoral lymphocytes were counted over 10 high power fields (hpf; field diameter of $0.55 \mathrm{~mm}$ ) in areas of highest density. The extent of intratumoral lymphocytes was designated as $1+$ (mild) if one to $<20$ lymphocytes were present in $10 \mathrm{hpf}, 2+$ (moderate) if 20-29 lymphocytes were present and
$3+$ (marked) if $\geq 30$ lymphocytes were counted. As a formal scoring system for intratumoral lymphocytes for urothelial carcinoma has not been previously proposed in the literature, these points were arbitrarily assigned (with guidance from scoring systems utilized in endometrial carcinomas ${ }^{45}$ ) before scoring. True tumor cell necrosis was documented as $1+$ if present in up to $5 \%$ of the tumor, $2+$ if present in $6-$ $50 \%$ and $3+$ if present in $>50 \%$ of the tumor.

Four $\mu$ m-thick tissue sections from a representative tumor block were subjected to immunohistochemical staining using anti-MLH-1 (clone G168.15, 1:20 in Van Gogh diluent; Biocare, Concord, CA, USA), anti-MSH-2 (clone FE11, 1:100 in Renoir Red Diluent; Biocare), anti-MSH-6 (clone BC/44, 1:100 in Renoir Red Diluent; Biocare), and antiPMS-2 (clone A16-4, ready to use, Biocare) utilizing a Leica Bond Polymer Refine DAB detection system, as previously described. ${ }^{50}$ Any degree of nuclear staining identified with appropriate staining of internal controls was considered positive. Loss of mismatch repair protein expression was defined by lack of nuclear staining in the lesional tissue, with appropriate internal controls. All H\&E and 
Table 1 Summary of patients included in Lynch syndrome category

\begin{tabular}{|c|c|c|c|c|c|}
\hline$P t^{\mathrm{a}}$ & Age & Gender & Common LAN & Other neoplasms & Germline status \\
\hline 2 & 53 & $\mathrm{~F}$ & CRC & Bladder UC & MSH2 mutation: IVS5+3A $>\mathrm{T}$ \\
\hline 3 & 66 & $\mathrm{~F}$ & CRC, EC & LG NET & $\begin{array}{l}\text { No personal genetic records } \\
\text { Son has } M S H 2 \text { mutation: IVS5+3A }>\mathrm{T}\end{array}$ \\
\hline 8 & 69 & M & SebN & ParACA, Bladder UC & MSH2 mutation: 420ins T \\
\hline 10 & 90 & M & CRC, AmpC & Bladder UC & $\begin{array}{l}\text { No genetic records; UTUC and CRC tested in-study with loss of MSH2/MSH6 } \\
\text { in both }\end{array}$ \\
\hline 11 & 60 & M & SebN & Bladder UC & No genetic records; Clinical MTS \\
\hline 13 & 57 & $\mathrm{M}$ & CRC & Bladder UC & MSH2 mutation: 421_422insT \\
\hline
\end{tabular}

Abbreviations: AmpC, ampullary carcinoma; CRC, colorectal carcinoma; EC, endometrial carcinoma; LAN, Lynch syndrome-associated neoplasm; LG NET, low-grade neuroendocrine tumor; MTS, Muir-Torre syndrome; ParACA, parotid adenocarcinoma; Pt, Patient; SebN, sebaceous neoplasms; UC, urothelial carcinoma; UTUC, upper tract urothelial carcinoma.

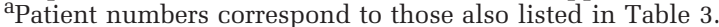

immunohistochemistry slides were reviewed by a single pathologist (HLH), with secondary review of select cases by at least one additional senior pathologist (CMG/TP). Review of morphology and immunohistochemistry results was carried out independently of each other and without knowledge of the patient's history.

\section{Results}

Of the total 444 patients with upper tract urothelial carcinoma, we analyzed a subset of 215 tumors from 214 patients (one patient had synchronous bilateral upper tract urothelial carcinoma), which included 194 consecutive cases (spanning a period from 2009 to 2014) and 21 additional cases specifically selected due to patient history of Lynch syndrome or Lynch syndrome-associated neoplasm. Our study population included 144 males and 70 females (male to female ratio of $\sim 2: 1$ ) with an average age of 70 years (range of 41-95 years). Thirty patients (14\%) had history of at least one additional common Lynch syndrome-associated neoplasm, including 20 with colorectal carcinoma, 4 with endometrial carcinoma, 2 with colorectal carcinoma and endometrial carcinoma, 2 with sebaceous neoplasms, 1 with ovarian carcinoma, and 1 with cholangiocarcinoma. Six patients were included in the Lynch syndrome category for statistical analysis and are summarized in Table 1. Three of these patients (2 with colorectal carcinoma and bladder urothelial carcinoma and 1 with bladder urothelial carcinoma and multiple sebaceous neoplasms) had documented germline mutations in MSH2 (c.IVS5+3A > T, c.421_422insT, and c.420insT, respectively). A fourth patient was presumed to have Lynch syndrome as she has history of colorectal carcinoma, endometrial carcinoma and small bowel neuroendocrine tumor and her son has Lynch syndrome with a documented germline mutation in MSH2 (c.IVS5+3A > T). A fifth patient with multiple sebaceous neoplasms had a history of Muir-Torre syndrome/Lynch syndrome, however documented only clinically in our medical record system. Finally, one additional patient was highly suspicious for having Lynch syndrome, as both his upper tract urothelial carcinoma and colorectal carcinoma demonstrated loss of MSH2/MSH6 by immunohistochemistry (both tested in-study). This patient also had a history of ampullary adenocarcinoma (no mismatch repair testing performed) and was included in the Lynch syndrome category (Table 1).

Eighty-four (39\%) upper tract urothelial carcinoma cases arose within the ureter, $101(47 \%)$ within the renal pelvis, and $30(14 \%)$ involved both locations. Papillary (predominantly exophytic) growth was noted in $78 \%$ of cases $(n=168)$, however 23 of these cases $(11 \%$ of cases overall) demonstrated inverted (endophytic) features, at least focally. No tumor was purely inverted/endophytic. Sixteen percent $(n=34)$ of tumors showed solid/nodular architecture and $6 \%$ $(n=13)$ were flat lesions. Tumor grade was distributed as follows: 179 (83\%) high-grade, 26 (12\%) lowgrade, and $10(5 \%)$ low-grade with focal high-grade features; $61(28 \%)$ tumors were stage $\mathrm{Ta}, 42(20 \%)$ T1, $27(13 \%)$ T2, $76(35 \%)$ T3 and $9(4 \%)$ stage T4 (Table 2). Necrosis was identified in $41 \%$ of cases $(24 \% 1+, 14 \% 2+$, and $3 \% 3+)$.

Loss of mismatch repair protein expression was identified in 14/215 (7\%) tumors (Table 3): 8 (57\%) involved the ureter, $4(29 \%)$ the renal pelvis, and 2 $(14 \%)$ involved both sites. Ten of these cases were identified among the non-selected group of 194 patients $(5 \%)$, with the remaining four occurring within the enriched group of 21 patients (19\%). The ages of patients with loss of mismatch repair protein expression ranged from 45 to 90 years (mean: 64); 9 were male, and 5 female. Twelve $(86 \%)$ cases with mismatch repair protein loss demonstrated loss of MSH2 and MSH6 (Figure 2), and 2 (14\%) showed isolated loss of MSH6 (Figure 3). All cases retained MLH1 and PMS2 expression. All cases with mismatch repair protein loss were high-grade (sensitivity $=100 \% ; \quad$ specificity $=18 \%$; odds ratio $=$ $>$ 999.999; $P$-value $=0.96$ ) without variant histology 
and $71 \%$ lacked nuclear pleomorphism (sensitivity = $71 \%$; specificity $=56 \%$; odds ratio $=3.1 ; P$-value $=$ 0.06; Figure 1c). Seven cases were pathologic stage $\mathrm{Ta} / \mathrm{T} 1$ and 7 T2/T3. Twenty-one percent $(3 / 14)$ of cases with mismatch repair protein loss displayed inverted growth features (Figure 1a), at least focally, compared with $10 \%(20 / 201)$ of cases with preserved

Table 2 Selected histopathologic characteristics of all tumors by anatomic site

\begin{tabular}{lrrrc}
\hline & $\begin{array}{r}\text { UUC } \\
\mathrm{n}=84\end{array}$ & $\begin{array}{c}\text { U+ RPUC } \\
\mathrm{n}=30\end{array}$ & $\begin{array}{c}\text { RPUC } \\
\mathrm{n}=101\end{array}$ & $\begin{array}{c}\text { Total } \\
\mathrm{n}=215(\%)\end{array}$ \\
\hline Grade & & & & \\
HG & 67 & 29 & 83 & $179(83)$ \\
LG & 14 & 0 & 12 & $26(12)$ \\
LG/HG & 3 & 1 & 6 & $10(5)$ \\
Stage & & & & \\
Ta & 25 & 3 & 33 & $61(28)$ \\
T1 & 12 & 7 & 23 & $42(20)$ \\
T2 & 16 & 2 & 9 & $27(13)$ \\
T3 & 30 & 17 & 29 & $76(35)$ \\
T4 & 1 & 1 & 7 & $9(4)$ \\
& & & & \\
Growth pattern & & & & \\
Papillary & & & & \\
$\quad$ Total & 59 & 25 & 84 & $168(78)$ \\
$\quad$ Exophytic & 49 & 25 & 71 & $145(67)$ \\
$\quad$ Inverted (focal) & 10 & 0 & 13 & $23(11)$ \\
Solid/nodule & 19 & 3 & 12 & $34(16)$ \\
Flat & 6 & 2 & 5 & $13(6)$ \\
Mismatch repair & & & & \\
protein-IHC loss & 8 & 2 & 4 & $14(7)$ \\
\hline
\end{tabular}

Abbreviations: HG, high-grade; LG, low-grade; LH/HG, low-grade with focal high-grade; RPUC, renal pelvis urothelial carcinoma; U+RPUC, ureteral plus renal pelvis urothelial carcinoma; UUC, ureteral urothelial carcinoma. mismatch repair protein expression (sensitivity $=$ $21 \%$; specificity $=90 \%$; odds ratio $=2.47 ; P$-value $=$ 0.19). Eighty-six percent of upper tract urothelial carcinoma with mismatch repair protein loss demonstrated pushing borders without destructive infiltrative edges (Figure 1b) compared to $53 \%$ of the cases with retained mismatch repair protein expression, a characteristic reaching statistical significance with a $P$-value of 0.03 (sensitivity $=86 \%$; specificity $=47 \%$; odds ratio $=5.4$ ). Utilizing ROC curve analysis of intratumoral lymphocyte counts, a cut-point of $2+$ ( $\geq 20$ lymphocytes/10 hpf) demonstrated the best balance of sensitivity and specificity (36 and 91\%, respectively) for evaluation and was statistically significant between the two groups with a $P$-value of 0.0045 (odds ratio $=5.65$ ). Moderate to marked $(2-3+)$ intratumoral lymphocytes (Figure 1d) were identified in 5 (36\%) upper tract urothelial carcinomas with loss of mismatch repair protein expression, compared to only $9 \%$ (18 of 201) of the cases with retained expression. Nine (64\%) of the 14 tumors with mismatch repair protein loss occurred in patients with other common Lynch syndromeassociated neoplasms, including the 6 patients with documented Lynch syndrome. Among the other three patients with history of Lynch syndromeassociated neoplasms, one patient had additional history of colorectal carcinoma and met Bethesda guidelines for suspicion of Lynch syndrome and two patients had history of endometrial carcinoma, however, none of these patients had available genetic records. Of the five patients without history of Lynch syndrome-associated neoplasm, one had a family history suspicious for inclusion under the Amsterdam criteria, having both a sister and daughter diagnosed with uterine carcinoma. However, the ages at diagnosis could not be identified to allow

Table 3 Characteristics of all tumors with mismatch repair protein-IHC loss

\begin{tabular}{|c|c|c|c|c|c|c|c|c|c|c|}
\hline Pt. & Site & Grade & Stage $(p T)$ & Border & Pleo. & Inv. & $\begin{array}{l}\text { ITL per } \\
10 \text { hpf }\end{array}$ & $\begin{array}{l}\text { Mismatch repair protein } \\
\text { lost by IHC }\end{array}$ & Common LAN & Other neoplasms \\
\hline 1 & $\mathrm{RP}$ & HG & 1 & $\mathrm{P}$ & - & + & 0 & MSH2/MSH6 & - & - \\
\hline 2 & $\mathrm{RP}$ & HG & a & $\mathrm{P}$ & - & + & 0 & MSH2/MSH6 & LS (CRC) & Bladder UC \\
\hline 3 & $\mathrm{RP}$ & HG & 2 & $\mathrm{P}$ & - & - & 0 & MSH2/MSH6 & LS (CRC, EC) & $\begin{array}{l}\text { Small bowel } \\
\text { LG NET }\end{array}$ \\
\hline 4 & $\mathrm{U}$ & HG & 2 & $\mathrm{P}$ & - & - & 23 & MSH2/MSH6 & 一 & SCC \\
\hline 5 & $\mathrm{U}$ & HG & 3 & I & + & - & 0 & MSH2/MSH6 & EC & - \\
\hline 6 & $\mathrm{U}$ & HG & a & $\mathrm{P}$ & + & - & 0 & MSH6 & - & Bladder UC \\
\hline 7 & $\mathrm{U}+\mathrm{RP}$ & HG & 3 & $\mathrm{P}$ & + & - & 0 & MSH2/MSH6 & $\mathrm{EC}$ & - \\
\hline 8 & $\mathrm{U}$ & HG & 1 & $\mathrm{P}$ & - & - & 35 & MSH2/MSH6 & LS/MTS (SebN) & ParACA, Bladder UC \\
\hline 9 & $\mathrm{U}+\mathrm{RP}$ & HG & 3 & $\mathrm{P}$ & - & - & 0 & MSH2/MSH6 & - & Bladder UC \\
\hline 10 & $\mathrm{U}$ & HG & 3 & $\mathrm{P}$ & - & - & 0 & MSH2/MSH6 & LS (CRC, AmpC) & Bladder UC \\
\hline 11 & $\mathrm{U}$ & HG & a & $\mathrm{P}$ & - & + & 3 & MSH2/MSH6 & LS/MTS (SebN) & Bladder UC \\
\hline 12 & $\mathrm{U}$ & HG & 3 & I & + & - & 31 & MSH2/MSH6 & CRC & - \\
\hline 13 & $\mathrm{U}$ & HG & a & $\mathrm{P}$ & - & - & 39 & MSH2/MSH6 & LS (CRC) & Bladder UC \\
\hline 14 & $\mathrm{RP}$ & HG & 1 & $\mathrm{P}$ & - & - & 35 & MSH6 & - & - \\
\hline
\end{tabular}

Abbreviations: a, stage pTa; AmpC, ampullary carcinoma; CRC, colorectal carcinoma; EC, endometrial carcinoma; HG, high-grade; I, infiltrative; Inv, inverted; ITL, intratumoral lymphocytes; LAN, Lynch syndrome-associated neoplasms; LG NET, low-grade neuroendocrine tumor; LS, Lynch syndrome; MTS, Muir-Torre syndrome; P, pushing; ParACA, parotid adenocarcinoma; Pleo, pleomorphism; RP, renal pelvis; SebN, sebaceous neoplasia; SCC, squamous cell carcinoma of skin; U, ureter; UC, urothelial carcinoma; U+RP, ureter and renal pelvis. 

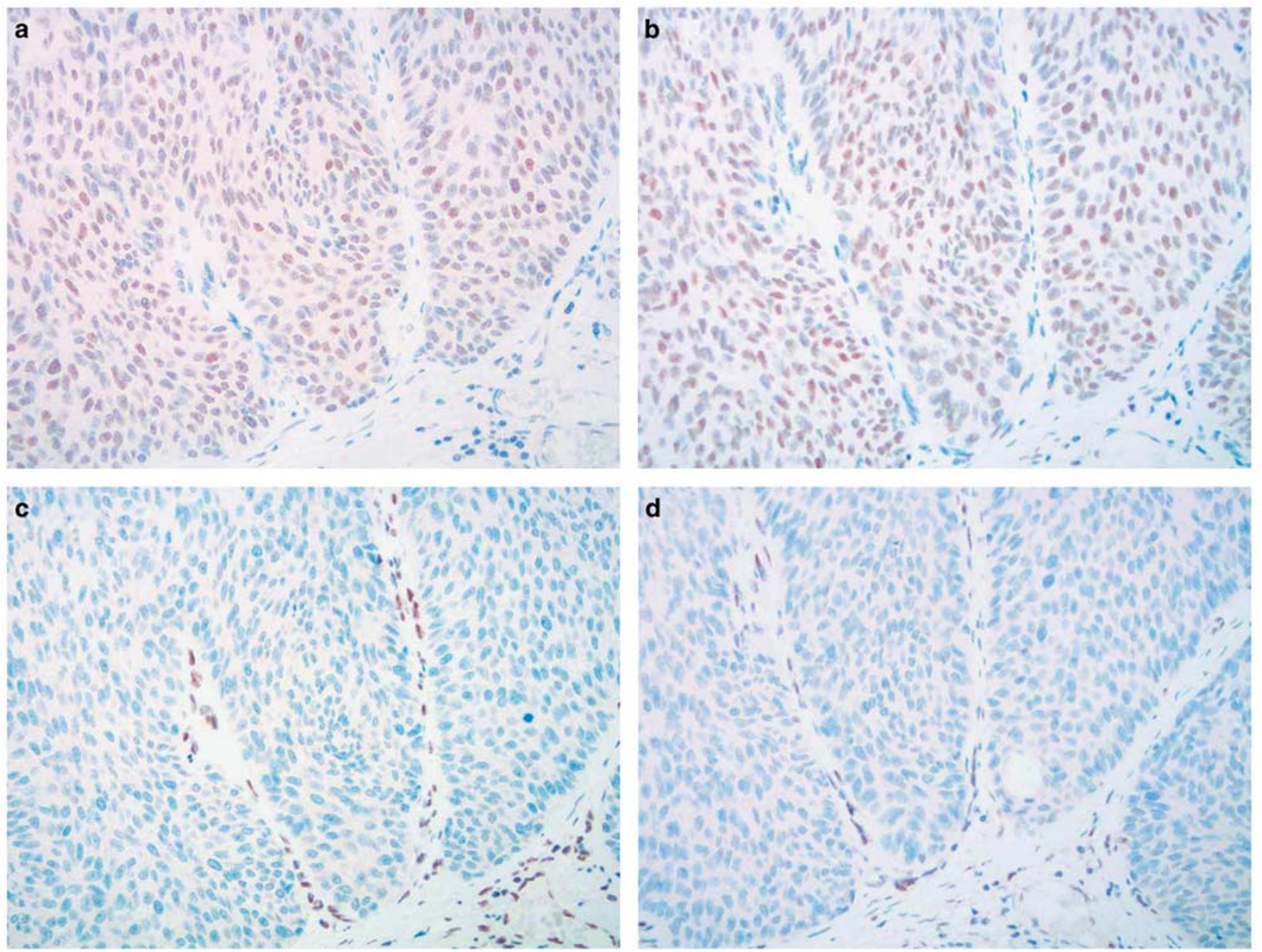

Figure 2 Example immunohistochemical profile in mismatch repair-deficient case demonstrating loss of MSH2 (c), and MSH6 (d) expression and retained expression of MLH1 (a) and PMS2 (b) in a patient with Muir-Torre syndrome.

definitive inclusion. The four remaining cases $(2 \%$ of unselected cases) with mismatch repair protein loss lacked documented history of any common Lynch syndrome-associated neoplasm and had no available genetic records.

All six upper tract urothelial carcinomas occurring in patients with Lynch syndrome demonstrated high-grade morphology, yet lacked significant pleomorphism and had pushing rather than infiltrative growth. Two cases demonstrated increased (at least moderate/2+) intratumoral lymphocytes (33\%) and two had inverted growth (33\%), at least focally (Table 3).

\section{Discussion}

Urothelial carcinoma of the upper urinary tract is becoming increasingly recognized as an extracolonic manifestation of Lynch syndrome. Upper tract urothelial carcinoma occurs in $5 \%$ of patients with Lynch syndrome, making it the third most common malignancy in this patient pop- ulation. ${ }^{5,13,32}$ As such, there is a growing literature exploring this association. ${ }^{13,14,17,27,33-35,38,51-56}$ To our knowledge, we report the largest series of predominantly unselected cases of upper tract urothelial carcinoma tested for mismatch repair protein loss by immunohistochemistry.

Lynch syndrome patients with upper tract urothelial carcinoma present at a younger age and often lack the typical risk factors associated with urothelial carcinoma in the general population. ${ }^{29,36}$ In our study, patients with mismatch repair-deficient upper tract urothelial carcinoma had an average age of 64 years, compared with that of 70 years in the overall cohort. The majority of these lesions occurred in the ureter $(57 \%)$, similar to findings reported by Hartmann et al. ${ }^{27}$

Patients suspected to have Lynch syndrome have historically been evaluated using the Amsterdam and Bethesda criteria; ${ }^{57-60}$ however, these criteria may fail to identify many patients at risk of having Lynch syndrome. ${ }^{24}$ As a result, pathology laboratories have been called on to improve detection through screening methodologies. In fact, universal 

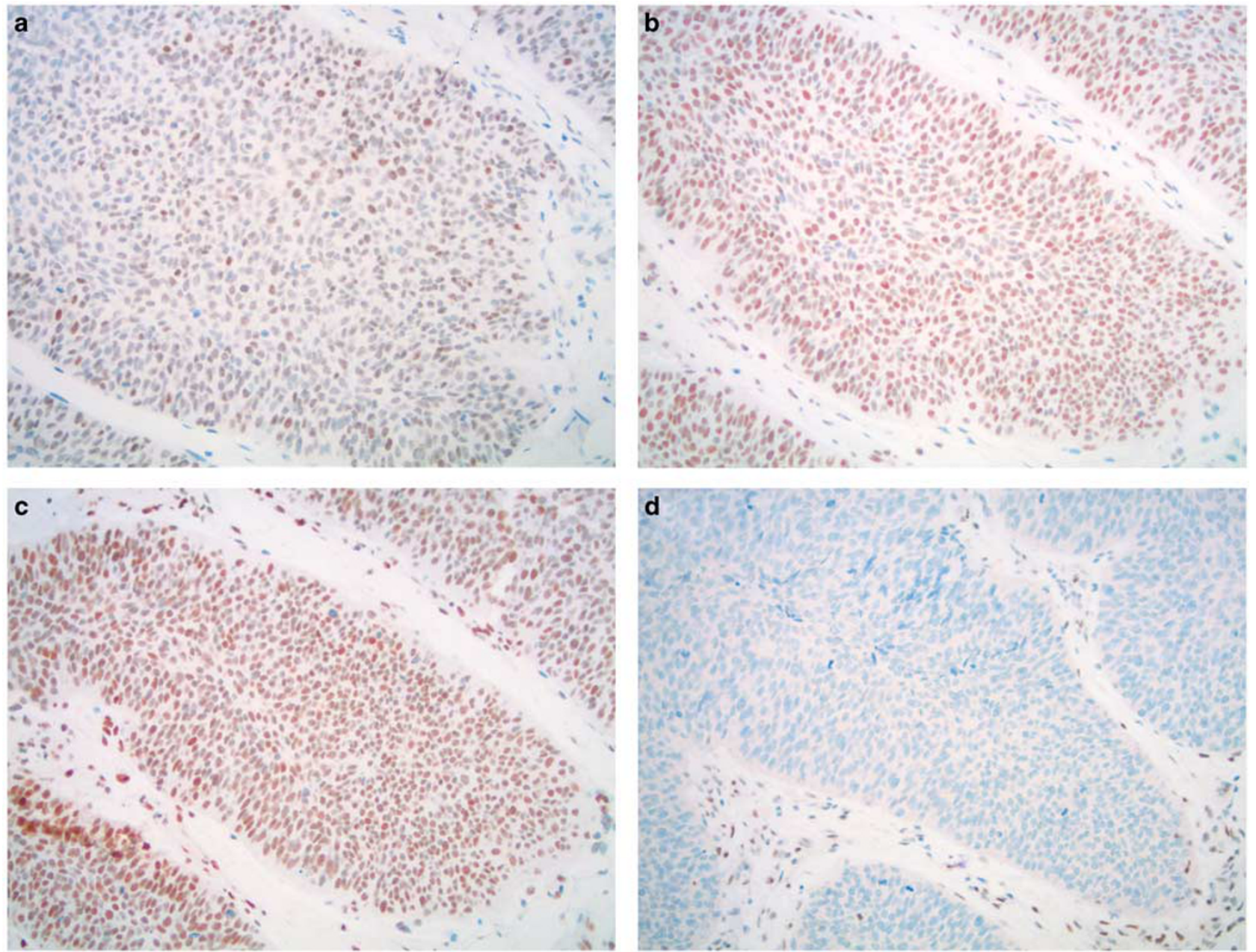

Figure 3 Example immunohistochemical profile in mismatch repair-deficient case demonstrating isolated loss of MSH6 (d) expression and retained expression of MLH1 (a), PMS2 (b), and MSH2 (c) in a patient without additional history of common Lynch syndromeassociated neoplasm.

screening of all colorectal carcinoma ${ }^{61,62}$ and endometrial carcinoma ${ }^{63-65}$ cases has been proposed and implemented in many institutions. Screening for defects in the mismatch repair system is typically achieved through microsatellite instability testing via polymerase chain reaction or through immunohistochemical staining for mismatch repair proteins. Although there has been debate as to which of these methods is superior, it is generally recognized that these tests are complimentary and both may be necessary in certain cases. ${ }^{62,66,67}$ However, both modalities are plagued by epigenetic silencing of mismatch repair genes via promoter hypermethylation, particularly of $M L H 1$, in a significant number of sporadic cases $(12 \%$ of upper tract urothelial carcinomas $^{52}$ and $10-15 \%$ of colorectal carcinoma $^{16,24}$ ). Further evaluation using BRAF (for colorectal carcinoma) or methylation testing can help resolve which of these cases are truly sporadic and which may be due to a germline mutation. ${ }^{16}$ More recent studies have also identified biallelic somatic mutations and loss of heterozygosity as the cause of aberrant microsatellite instability and mismatch repair protein immunohistochemistry testing in a subset of patients, ${ }^{68-71}$ highlighting the need for confirmatory germline mutation analysis in patients with apparent mismatch repair defects. In addition, specific cancer risks in patients with confirmed Lynch syndrome are strongly influenced by which mismatch repair gene is mutated, ${ }^{21}$ so identification of the specific mutation should be sought to guide patient screening and follow-up, and to direct identification of additional affected family members.

Microsatellite instability was first described in upper tract urothelial carcinoma in $1998 ;^{29}$ since then, high-level microsatellite instability has been documented in $4-31 \%$ of 'sporadic' upper tract urothelial carcinoma cases. . $^{2,38,51,54,55,72}$ Having identified mismatch repair protein loss in $7 \%$ of the 215 upper tract urothelial carcinomas we evaluated, the incidence of a mismatch repair defect in our series is on the lower end of the reported incidence range. However, it is worth mentioning 
that the majority of prior studies analyzed microsatellite instability using polymerase chain reaction with wide variability in the microsatellite markers utilized and in how the authors defined high microsatellite instability itself. ${ }^{29}$

Urothelial carcinoma is more likely to develop in patients with germline mutations in MSH2. In support of this notion, $86 \%$ of the cases demonstrating mismatch repair protein loss in our series lacked staining for both MSH2 and MSH6, suggesting an underlying MSH2 mutation. The remaining 14\% have a high likelihood of harboring a germline mutation in either $\mathrm{MSH} 6$ or its partner, $\mathrm{MSH}$, as evidenced by loss of MSH6 protein expression. As prior studies have reported that immunohistochemical loss of MSH2 or MSH6 is frequently associated with an underlying germline mutation, ${ }^{16,20,24,37,73,74}$ testing of upper tract urothelial carcinoma may prove to have higher yield of true mutations than colorectal carcinoma or endometrial carcinoma screening.

Interestingly, we identified mismatch repair protein loss by immunohistochemistry in $30 \%$ of the patients with common Lynch syndrome-associated neoplasms, and, in fact, the majority (64\%) of patients with mismatch repair-deficient upper tract urothelial carcinoma had previous history of an additional Lynch syndrome-associated neoplasm, most commonly colorectal carcinoma. In addition, upper tract urothelial carcinoma from all six patients with Lynch syndrome demonstrated mismatch repair protein loss, suggesting that immunohistochemistry may be adequately sensitive for use in screening for mismatch repair defects in this population. Although all mismatch repair proteinnegative cases showed loss of MSH2/MSH6 or MSH6, both of which are frequently associated with an underlying germline mutation, ${ }^{16,20,24,37,73,74}$ it is more difficult to comment on the specificity of immunohistochemistry in our series as the germline status of all patients is not known.

Although it is less likely than colorectal carcinoma or endometrial carcinoma to be the sentinel lesion, upper tract urothelial carcinoma may be the presenting malignancy in a small number of Lynch syndrome patients. We identified loss of mismatch repair protein expression in $2.7 \%(5 / 184)$ of the patients without history of any additional Lynch syndrome-associated neoplasm. As such, identifying morphologic characteristics common to mismatch repair-deficient upper tract urothelial carcinoma may prove helpful in identifying patients without history of Lynch syndrome-associated neoplasms that may benefit from further testing. Several previous reports have suggested inverted growth pattern, pushing borders, and low tumor stage as features suggestive of mismatch repair deficiency. ${ }^{13,14,27,35}$ Additional studies have examined the degree of inflammatory response ${ }^{14,27}$ and tumor grade. ${ }^{13,14,27,34,35,38,51}$ The results of these studies have been quite varied, and consistently reproducible morphologic features have not yet been defined. We also sought to identify unifying features among our cases demonstrating mismatch repair protein loss by immunohistochemistry. Lack of nuclear pleomorphism (sensitivity $=71 \%$; specificity $=56 \%$; odds ratio $=3.1 ; P$-value $=0.06$ ), inverted growth pattern (sensitivity $=21 \%$; specificity $90 \%$; odds ratio $=2.47 ; P$-value $=0.19$ ) presence of pushing borders without destructive infiltrative edges (sensitivity $=86 \%$; specificity $=47 \%$; odds ratio $=$ 5.4 ) and increased intratumoral lymphocytes (sensitivity $=36 \%$; specificity $=91 \%$; odds ratio $=5.65$ ) emerged as features which may help identify potential Lynch syndrome candidates. Among these characteristics, increased intratumoral lymphocytes ( $\geq 20 \mathrm{TIL} / 10 \mathrm{hpf}$ ) and pushing tumor/stromal interface reached statistical significance with $P$-values of 0.0045 and 0.03 , respectively. It is clear that no one morphologic feature is sensitive or specific enough to suggest Lynch syndrome with certainty, but that these features may need to be considered as a constellation of findings when contemplating further analysis for mismatch repair defects.

To our knowledge, the findings reported herein represent the largest series of predominantly unselected upper tract urothelial carcinomas assessed for mismatch repair defects via immunohistochemistry. Our exploration of histopathologic features noted among mismatch repair-deficient tumors failed to identify any with the degree of sensitivity needed for screening, but does suggest that the presence of pushing borders and increased intratumoral lymphocytes should prompt consideration of additional testing. Our data also suggests that immunohistochemistry has sufficient sensitivity to serve as a screening tool for underlying mismatch repair defects. In fact, it appears as though limited assessment for MSH2 and MSH6 only may have more utility for screening in suspicious cases than assessment of all four mismatch repair proteins. We also provide additional evidence that we hope will help guide future discussions regarding the appropriateness of universal screening in upper tract urothelial carcinoma. Our data strongly suggest that special consideration for additional testing should at least be paid to younger patients and those with history of other common Lynch syndrome-associated neoplasms.

\section{Acknowledgments}

We would like to thank Karen Streator Smith for her dedication, support and tremendous contribution in performing many of the technical aspects required to complete this study.

\section{Disclosure/conflict of interest}

Brandie Heald serves on the speakers' bureau for Myriad Genetics Lab and on the advisory board for Invitae. The remaining authors declare no conflict of interest. 


\section{References}

1 Siegel RL, Miller KD, Jemal A. Cancer statistics, 2016. CA Cancer J Clin 2016;66:7-30.

2 Huben RP, Mounzer AM, Murphy GP. Tumor grade and stage as prognostic variables in upper tract urothelial tumors. Cancer 1988;62:2016-2020.

3 David KA, Mallin K, Milowsky MI, et al. Surveillance of urothelial carcinoma: stage and grade migration, 1993-2005 and survival trends, 1993-2000. Cancer 2009;115:1435-1447.

4 Raman JD, Messer J, Sielatycki JA, et al. Incidence and survival of patients with carcinoma of the ureter and renal pelvis in the USA, 1973-2005. BJU Int 2011;107: 1059-1064.

5 Yates DR, Catto JW. Distinct patterns and behaviour of urothelial carcinoma with respect to anatomical location: how molecular biomarkers can augment clinicopathological predictors in upper urinary tract tumours. World J Urol 2013;31:21-29.

6 Roupret M, Babjuk M, Comperat E, et al. European Association of urology guidelines on upper urinary tract urothelial cell carcinoma: 2015 update. Eur Urol 2015;68:868-879.

7 Hall MC, Womack S, Sagalowsky AI, et al. Prognostic factors, recurrence, and survival in transitional cell carcinoma of the upper urinary tract: a 30-year experience in 252 patients. Urology 1998;52:594-601.

8 Lughezzani G, Burger M, Margulis V, et al. Prognostic factors in upper urinary tract urothelial carcinomas: a comprehensive review of the current literature. Eur Urol 2012;62:100-114.

9 Patel N, Arya M, Muneer A, et al. Molecular aspects of upper tract urothelial carcinoma. Urol Oncol 2014;32: e20.

10 Hubosky SG, Boman BM, Charles S, et al. Ureteroscopic management of upper tract urothelial carcinoma (UTUC) in patients with Lynch syndrome (hereditary nonpolyposis colorectal cancer syndrome). BJU Int 2013;112:813-819.

11 Watson P, Lynch HT. Extracolonic cancer in hereditary nonpolyposis colorectal cancer. Cancer 1993;71: 677-685.

12 Watson P, Lynch HT. The tumor spectrum in HNPCC. Anticancer Res 1994;14:1635-1639.

13 Garcia-Tello A, Ramon de Fata F, Andres G, et al. DNA repair genes and prognosis in sporadic forms of urothelial carcinoma of the upper urinary tract. Actas Urol Esp 2014;38:600-607.

14 Hartmann A, Dietmaier W, Hofstadter F, et al. Urothelial carcinoma of the upper urinary tract: inverted growth pattern is predictive of microsatellite instability. Hum Pathol 2003;34:222-227.

15 Mongiat-Artus P, Miquel C, Flejou JF, et al. Spectrum of molecular alterations in colorectal, upper urinary tract, endocervical, and renal carcinomas arising in a patient with hereditary non-polyposis colorectal cancer. Virchows Arch 2006;449:238-243.

16 Bhalla A, Zulfiqar M, Weindel M, et al. Molecular diagnostics in colorectal carcinoma. Clin Lab Med 2013;33:835-859.

17 Skeldon SC, Semotiuk K, Aronson M, et al. Patients with Lynch syndrome mismatch repair gene mutations are at higher risk for not only upper tract urothelial cancer but also bladder cancer. Eur Urol 2013;63: $379-385$.
18 Eltz S, Comperat E, Cussenot O, et al. Molecular and histological markers in urothelial carcinomas of the upper urinary tract. BJU Int 2008;102:532-535.

19 Lynch HT, Snyder CL, Shaw TG, et al. Milestones of Lynch syndrome: 1895-2015. Nat Rev Cancer 2015;15: 181-194.

20 Giardiello FM, Allen JI, Axilbund JE, et al. Guidelines on genetic evaluation and management of Lynch syndrome: a consensus statement by the US Multisociety Task Force on colorectal cancer. Am J Gastroenterol 2014;109:1159-1179.

21 Cohen SA, Leininger A. The genetic basis of Lynch syndrome and its implications for clinical practice and risk management. Appl Clin Genet 2014;7: 147-158.

22 Rhees J, Arnold M, Boland CR. Inversion of exons 1-7 of the MSH2 gene is a frequent cause of unexplained Lynch syndrome in one local population. Fam Cancer 2014;13:219-225.

23 Wagner A, van der Klift H, Franken P, et al. A 10- Mb paracentric inversion of chromosome arm $2 p$ inactivates MSH2 and is responsible for hereditary nonpolyposis colorectal cancer in a North-American kindred. Genes Chromosomes Cancer 2002;35:49-57.

24 Newton K, Jorgensen NM, Wallace AJ, et al. Tumour MLH1 promoter region methylation testing is an effective prescreen for Lynch syndrome (HNPCC). J Med Genet 2014;51:789-796.

25 Woerner SM, Benner A, Sutter C, et al. Pathogenesis of DNA repair-deficient cancers: a statistical metaanalysis of putative real common target genes. Oncogene 2003;22:2226-2235.

26 Duval A, Reperant M, Compoint A, et al. Target gene mutation profile differs between gastrointestinal and endometrial tumors with mismatch repair deficiency. Cancer Res 2002;62:1609-1612.

27 Hartmann A, Zanardo L, Bocker-Edmonston T, et al. Frequent microsatellite instability in sporadic tumors of the upper urinary tract. Cancer Res 2002;62: 6796-6802.

28 Mork M, Hubosky SG, Roupret M, et al. Lynch Syndrome: A Primer for Urologists and Panel Recommendations. J Urol 2015;194:21-29.

29 Roupret M, Yates DR, Comperat E, et al. Upper urinary tract urothelial cell carcinomas and other urological malignancies involved in the hereditary nonpolyposis colorectal cancer (lynch syndrome) tumor spectrum. Eur Urol 2008;54:1226-1236.

30 Boennelycke M, Thomsen BM, Holck S. Sebaceous neoplasms and the immunoprofile of mismatch-repair proteins as a screening target for syndromic cases. Pathol Res Pract 2015;211:78-82.

31 Bhaijee F, Brown AS. Muir-Torre syndrome. Arch Pathol Lab Med 2014;138:1685-1689.

32 Ericson K, Halvarsson B, Nagel J, et al. Defective mismatch-repair in patients with multiple primary tumours including colorectal cancer. Eur J Cancer 2003;39:240-248.

33 van der Post RS, Kiemeney LA, Ligtenberg MJ, et al. Risk of urothelial bladder cancer in Lynch syndrome is increased, in particular among MSH2 mutation carriers. J Med Genet 2010;47:464-470.

34 Bai S, Nunez AL, Wei S, et al. Microsatellite instability and TARBP2 mutation study in upper urinary tract urothelial carcinoma. Am J Clin Pathol 2013;139: $765-770$. 
35 Crockett DG, Wagner DG, Holmang S, et al. Upper urinary tract carcinoma in Lynch syndrome cases. J Urol 2011;185:1627-1630.

36 Sijmons RH, Kiemeney LA, Witjes JA, et al. Urinary tract cancer and hereditary nonpolyposis colorectal cancer: risks and screening options. J Urol 1998;160: 466-470.

37 Hartmann A, Cheville JC, Dietmaier W, et al. Hereditary nonpolyposis colorectal cancer syndrome in a patient with urothelial carcinoma of the upper urothelial tract. Arch Pathol Lab Med 2003;127:E60-E63.

38 Ericson KM, Isinger AP, Isfoss BL, et al. Low frequency of defective mismatch repair in a population-based series of upper urothelial carcinoma. BMC cancer 2005;5:23.

39 Catto JW, Azzouzi AR, Amira N, et al. Distinct patterns of microsatellite instability are seen in tumours of the urinary tract. Oncogene 2003;22:8699-8706.

40 Geary J, Sasieni P, Houlston R, et al. Gene-related cancer spectrum in families with hereditary nonpolyposis colorectal cancer (HNPCC). Fam Cancer 2008;7:163-172.

41 Watson P, Vasen HF, Mecklin JP, et al. The risk of extra-colonic, extra-endometrial cancer in the Lynch syndrome. Int J Cancer 2008;123:444-449.

42 Shia J, Holck S, Depetris G, et al. Lynch syndromeassociated neoplasms: a discussion on histopathology and immunohistochemistry. Fam Cancer 2013;12: 241-260.

43 Shia J, Ellis NA, Paty PB, et al. Value of histopathology in predicting microsatellite instability in hereditary nonpolyposis colorectal cancer and sporadic colorectal cancer. Am J Surg Pathol 2003;27:1407-1417.

44 Garg K, Leitao MM Jr., Kauff ND, et al. Selection of endometrial carcinomas for DNA mismatch repair protein immunohistochemistry using patient age and tumor morphology enhances detection of mismatch repair abnormalities. Am J Surg Pathol 2009;33: 925-933.

45 Shia J, Black D, Hummer AJ, et al. Routinely assessed morphological features correlate with microsatellite instability status in endometrial cancer. Hum Pathol 2008;39:116-125.

46 Garg K, Soslow RA. Lynch syndrome (hereditary nonpolyposis colorectal cancer) and endometrial carcinoma. J Clin Pathol 2009;62:679-684.

47 Chui MH, Ryan P, Radigan J, et al. The histomorphology of Lynch syndrome-associated ovarian carcinomas: toward a subtype-specific screening strategy. Am J Surg Pathol 2014;38:1173-1181.

48 Eble JN, Sauter G, Epstein JI, et al. World Health Organization Classification of Tumours. Pathology and Genetics of Tumours of the Urinary System and Male Genital Organs. IARC Press: Lyon, France, 2004.

49 Mostofi F, Sobin L. Histologic Typing of Urinary Bladder Tumors. World Health Organization: Geneva, 1973.

50 Morrison J, Bronner M, Leach BH, et al. Lynch syndrome screening in newly diagnosed colorectal cancer in general pathology practice: from the revised Bethesda guidelines to a universal approach. Scand J Gastroenterol 2011;46:1340-1348.

51 Blaszyk H, Wang L, Dietmaier W, et al. Upper tract urothelial carcinoma: a clinicopathologic study including microsatellite instability analysis. Mod Pathol 2002;15:790-797.
52 Catto JW, Azzouzi AR, Rehman I, et al. Promoter hypermethylation is associated with tumor location, stage, and subsequent progression in transitional cell carcinoma. J Clin Oncol 2005;23:2903-2910.

53 Mongiat-Artus $\mathrm{P}$, Miquel C, Van der Aa $\mathrm{M}$, et al. Microsatellite instability and mutation analysis of candidate genes in urothelial cell carcinomas of upper urinary tract. Oncogene 2006;25:2113-2118.

54 Roupret M, Catto J, Coulet F, et al. Microsatellite instability as indicator of $\mathrm{MSH} 2$ gene mutation in patients with upper urinary tract transitional cell carcinoma. J Med Genet 2004;41:e91.

55 Roupret M, Coulet F, Azzouzi AR, et al. Accuracy of the routine detection of mutation in mismatch repair genes in patients with susceptibility to hereditary upper urinary tract transitional cell carcinoma. BJU Int 2005;96:149-151.

56 Roupret M, Fromont G, Azzouzi AR, et al. Microsatellite instability as predictor of survival in patients with invasive upper urinary tract transitional cell carcinoma. Urology 2005;65:1233-1237.

57 Vasen HF, Mecklin JP, Khan PM, et al. The International Collaborative Group on hereditary non-polyposis colorectal cancer (ICG-HNPCC). Dis Colon Rectum 1991;34:424-425.

58 Vasen HF, Watson P, Mecklin JP, et al. New clinical criteria for hereditary nonpolyposis colorectal cancer (HNPCC, Lynch syndrome) proposed by the International Collaborative group on HNPCC. Gastroenterology 1999;116:1453-1456.

59 Boland CR, Thibodeau SN, Hamilton SR, et al. A National Cancer Institute Workshop on Microsatellite Instability for cancer detection and familial predisposition: development of international criteria for the determination of microsatellite instability in colorectal cancer. Cancer Res 1998;58:5248-5257.

60 Umar A, Boland CR, Terdiman JP, et al. Revised Bethesda Guidelines for hereditary nonpolyposis colorectal cancer (Lynch syndrome) and microsatellite instability. J Natl Cancer Inst 2004;96:261-268.

61 Musulen E, Sanz C, Munoz-Marmol AM, et al. Mismatch repair protein immunohistochemistry: a useful population screening strategy for Lynch syndrome. Hum Pathol 2014;45:1388-1396.

62 Group EoGAiPPW. Recommendations from the EGAPP Working Group: genetic testing strategies in newly diagnosed individuals with colorectal cancer aimed at reducing morbidity and mortality from Lynch syndrome in relatives. Genet Med 2009;11:35-41.

63 Frolova AI, Babb SA, Zantow E, et al. Impact of an immunohistochemistry-based universal screening protocol for Lynch syndrome in endometrial cancer on genetic counseling and testing. Gynecologic oncology 2015;137:7-13.

64 Mills AM, Longacre TA. Lynch syndrome screening in the gynecologic tract: current state of the art. Am J Surg Pathol 2016;40:e35-e44.

65 Mills AM, Sloan EA, Thomas M, et al. Clinicopathologic comparison of Lynch syndrome-associated and "lynch-like" endometrial carcinomas identified on universal screening using mismatch repair protein immunohistochemistry. Am J Surg Pathol 2016;40: 155-165.

66 Funkhouser WK Jr., Lubin IM, Monzon FA, et al. Relevance, pathogenesis, and testing algorithm for mismatch repair-defective colorectal carcinomas: a 
report of the association for molecular pathology. J Mol Diagn 2012;14:91-103.

67 Goodfellow PJ, Billingsley CC, Lankes HA, et al. Combined microsatellite instability, MLH1 methylation analysis, and immunohistochemistry for Lynch syndrome screening in endometrial cancers from GOG210: an NRG Oncology and Gynecologic Oncology Group Study. J Clin Oncol 2015;33:4301-4308.

68 Kang SY, Park CK, Chang DK, et al. Lynch-like syndrome: characterization and comparison with EPCAM deletion carriers. Int J Cancer 2015;136: 1568-1578.

69 Geurts-Giele WR, Leenen CH, Dubbink HJ, et al. Somatic aberrations of mismatch repair genes as a cause of microsatellite-unstable cancers. J Pathol 2014;234:548-559.

70 Haraldsdottir S, Hampel H, Tomsic J, et al. Colon and endometrial cancers with mismatch repair deficiency can arise from somatic, rather than germline, mutations. Gastroenterology 2014;147:1308-1316.

71 Mensenkamp AR, Vogelaar IP, van Zelst-Stams WA, et al. Somatic mutations in MLH1 and MSH2 are a frequent cause of mismatch-repair deficiency in Lynch syndromelike tumors. Gastroenterology 2014;146:643-646.

72 Amira N, Rivet J, Soliman H, et al. Microsatellite instability in urothelial carcinoma of the upper urinary tract. J Urol 2003;170:1151-1154.

73 Gibson J, Lacy J, Matloff E, et al. Microsatellite instability testing in colorectal carcinoma: a practical guide. Clin Gastroenterol Hepatol 2014;12:171-176.

74 Dudley B, Brand RE, Thull D, et al. Germline MLH1 mutations are frequently identified in Lynch syndrome patients with colorectal and endometrial carcinoma demonstrating isolated loss of PMS2 immunohistochemical expression. Am J Surg Pathol 2015;39: 1114-1120. 